Département de sciences économiques

Cahier 2001-27

\title{
Probabilistic Assignments of Identical Indivisible Objects and Uniform Probabilistic Rules
}

EHLERS, Lars

KLAUS, Bettina 


\section{Département de sciences économiques}

Université de Montréal

Faculté des arts et des sciences

C.P. 6128, succursale Centre-Ville

Montréal (Québec) H3C 3J7

Canada

http://www.sceco.umontreal.ca

SCECO-information@UMontreal.CA

Téléphone : (514) 343-6539

Télécopieur : (514) 343-7221

Ce cahier a également été publié par le Centre interuniversitaire de recherche en économie quantitative (CIREQ) sous le numéro 27-2001.

This working paper was also published by the Center for Interuniversity Research in Quantitative Economics (CIREQ), under number 27-2001.

ISSN 0709-9231 


\title{
CAHIER 2001-27
}

\section{PROBABILISTIC ASSIGNMENTS OF IDENTICAL INDIVISIBLE OBJECTS AND UNIFORM PROBABILISTIC RULES}

\author{
Lars EHLERS ${ }^{1}$ and Bettina KLAUS ${ }^{2}$
}

1 Centre de recherche et développement en économique (C.R.D.E.) and Département de sciences économiques, Université de Montréal

2 Department of Economics, University of Nebraska at Lincoln

October 2001

The authors thank William Thomson for many helpful comments. 


\title{
RÉSUMÉ
}

Nous considérons une approche probabiliste au problème de répartition de $k$ objets identiques à un ensemble d'agents avec des préférences unimodales. Utilisant l'extension ordinale des préférences, nous caractérisons les classes de lois de probabilités uniformes par l'efficacité au sens de Pareto, la non-manipulation et l'absence d'envie. Nous montrons aussi que l'anonymat ne peut pas être remplacé par l'absence d'envie. Quand les agents ont une fonction d'utilité Von-Neumann-Morgenstern strictement adverse au risque, nous ramenons le problème de répartition de $k$ objets identiques au problème d'allocation d'une quantité $k$ d'un bien infiniment divisible.

Mots clés: lois de probabilité, préférences unimodales, absence de manipulation, allocation uniforme

\begin{abstract}
We consider a probabilistic approach to the problem of assigning $k$ indivisible identical objects to a set of agents with single-peaked preferences. Using the ordinal extension of preferences, we characterize the class of uniform probabilistic rules by Pareto efficiency, strategy-proofness, and no-envy. We also show that in this characterization no-envy cannot be replaced by anonymity. When agents are strictly risk averse von-Neumann-Morgenstern utility maximizers, then we reduce the problem of assigning $k$ identical objects to a problem of allocating the amount $k$ of an infinitely divisible commodity.
\end{abstract}

Key words : probabilistic rules, single-peaked preferences, strategy-proofness, uniform allocations 


\section{Introduction}

We consider economic environments where a set of indivisible identical objects have to be assigned to a set of individuals with single-peaked preferences. As an example, take the assignment of the number of courses a professor in an economics department has to teach. Each professor has a number of courses he finds optimal (probably somewhere between 0 to 4) and preferences are decreasing when moving away from that optimal amount in either direction. If we are interested in "fair allocations", for instance allocations that respect equal treatment of equals (if two individuals have the same preference relation, then they should be indifferent between each other's allotments), then the indivisibility assumption may induce an impossibility. For simplicity, assume that we have to assign a course and none of the professors in the department wants to teach it. Obviously there is no deterministic way to do so that respects equal treatment of equals. However, allowing the rule to be probabilistic solves our problem at least in an ex-ante sense: if each professor has to teach the course with equal probability, then equal treatment of equals is satisfied (ex-ante).

The probabilistic allocation or rationing of indivisible objects has received recent attention. Two main models should be distinguished. In the first one there are $n$ objects and $n$ agents and each agent receives exactly one object. Any two objects are distinct and each agent has a strict preference relation over the set of objects. For example, the agents are workers and each object is a full-time job at a different company. The random assignment of the objects to the agents is the subject of papers by Abdulkadiroğlu and Sönmez (1998, 2000), Bogomolnaia and Moulin (1999), and Crès and Moulin (1998).

In the second model there are $k$ identical indivisible objects and $n$ agents. Each agent receives a certain number of objects and each object is assigned to some agent (free disposal is not allowed). For instance, the objects are identical (non full-time) jobs that have to be allocated among workers. This model is studied by Moulin (2000), Moulin and Stong (2000), Sasaki (1997), and Kureishi (2000). In the first two papers each agent demands a certain number of objects and the total demand is greater than the number of objects available. In the last two papers each agent has a single-peaked preference relation over the number of objects he may receive. That is, there is a most preferred number of objects, called the agent's peak, and preferences are strictly decreasing in either direction away from the peak. A probabilistic rule chooses for each profile of preferences a probability distribution over the set of allocations. The interpretation is that the final allocation that we implement is drawn according to this distribution. An agent compares two distributions over the set of allocations by evaluating the marginal distributions that are induced over his allotments. 
We consider the same model as Sasaki (1997) and Kureishi (2000), but we do not only consider preferences that can be represented by von-Neumann-Morgenstern utility functions. First, we use the ordinal extension of preferences over allotments to probability distributions over allotments. An agent prefers a distribution over his allotments to another if the first distribution places on each weak upper contour set at least the same probability that is placed on it by the second distribution. The ordinal extension of preferences is incomplete over the set of distributions. However, it is equivalent to the following. If an agent prefers one distribution to another, then for each utility representation of his preference relation the expected utility with respect to the distribution is greater than or equal to the expected utility of the second one. ${ }^{1}$

Using this extension we formulate the requirements of Pareto efficiency, strategy-proofness (no agent can gain by misrepresenting his preference relation), and no-envy (each agent prefers his marginal distribution to each other agent's marginal distribution). Contrary to the model with distinct objects (Bogomolnaia and Moulin, 1999) it turns out that in our model Pareto efficiency is equivalent to ex-post efficiency. Using the uniform rule (Benassy, 1982), where $k$ units of an infinitely divisible commodity are rationed as equally as possible, we define uniform probabilistic rules (Sasaki, 1997) in terms of their "uniform marginal distributions". ${ }^{2}$

We call a probabilistic rule a uniform probabilistic rule if for each profile the marginals of the chosen distribution are equal to the uniform marginal distributions at this profile. Our main result is that the class of uniform probabilistic rules is characterized by Pareto efficiency, strategy-proofness, and no-envy. This result is the probabilistic analogue to the result of Sprumont (1991). He shows that when rationing $k$ units of an infinitely divisible commodity among a set of individuals with single-peaked preferences, the uniform rule is the only deterministic rule satisfying the above combination of properties. Sprumont's characterization remains valid if we replace no-envy by anonymity (Sprumont, 1991) or equal treatment of equals (Ching, 1994). However, in our probabilistic setting this conclusion is not true. For example, any random dictatorship rule satisfies Pareto efficiency, strategy-proofness, and anonymity. It is an open question what the class of probabilistic rules satisfying these properties looks like.

\footnotetext{
${ }^{1}$ A considerable number of papers considers the ordinal extension of preferences, e.g., Abdulkadiroğlu and Sönmez (2000), Bogomolnaia and Moulin (1999), Ehlers (1998), Ehlers and Klaus (2001), Ehlers, Peters, and Storcken (2000), and Gibbard (1977).

${ }^{2}$ Independently, Moulin (2000) also used this trick in defining the uniform probabilistic rule of Sasaki (1997).
} 
In two related papers (Sasaki, 1997; Kureishi, 2000) agents are assumed to be strictly risk averse von-Neumann-Morgenstern utility maximizer, i.e., each agent evaluates distributions on the basis of the expected utility relative to his utility function. They show that for a given profile of utility functions, if a distribution is Pareto efficient, then each agent's marginal distribution places probability 1 on two allotments that differ only by one unit. Using this observation we show that then, the problem can be reduced to the problem of allocating $k$ units of an infinitely divisible commodity among $n$ agents with single-peaked preferences over $[0, k]$. Then we apply the characterization of the (deterministic) uniform rule by Ching (1994) and show that in the probabilistic model with strictly risk averse agents, the class of uniform probabilistic rules is characterized by Pareto efficiency, strategy-proofness, and equal treatment of equals. Therefore, the results of Sasaki (1997) and Kureishi (2000) can be interpreted as corollaries of Ching (1994).

The organization of the paper is as follows. In Section 2 we introduce the model and basic properties. In Section 3 we define the class of uniform probabilistic rules and present our main result. In Section 4 we prove the characterization. Finally, in Section 5 we focus on strictly risk averse agents with von-Neumann-Morgenstern utility functions.

\section{The Model and Basic Properties}

We consider the problem of assigning $k$ indivisible identical objects to a set of agents $N=\{1, \ldots, n\}$. Each agent $i \in N$ is equipped with a "single-peaked" preference relation $R_{i}$ defined over the number of objects he receives; i.e., $R_{i}$ is defined over $K \equiv\{0,1, \ldots, k\}$ and there exists a number of objects $p\left(R_{i}\right) \in K$, called the peak of $R_{i}$, with the following property: for all $x_{i}, y_{i} \in K$, if $x_{i}<y_{i} \leq p\left(R_{i}\right)$ or $x_{i}>y_{i} \geq p\left(R_{i}\right)$, then $y_{i} P_{i} x_{i}$. As usual, $x_{i} R_{i} y_{i}$ means " $x_{i}$ is weakly preferred to $y_{i}$ ", and $x_{i} P_{i} y_{i}$ means " $x_{i}$ is strictly preferred to $y_{i}$ ". By $\mathcal{R}$ we denote the class of all single-peaked preference relations over $K$. By $\mathcal{R}^{N}$ we denote the set of all (preference) profiles $R=\left(R_{i}\right)_{i \in N}$ such that for all $i \in N, R_{i} \in \mathcal{R}$.

We call $x \in K^{N}$ a feasible allocation if $\sum_{i \in N} x_{i}=k$. Let $X$ denote the set of all feasible allocations. Note that $|X|=\left(\begin{array}{c}k+n-1 \\ k\end{array}\right)$. A deterministic (allocation) rule $\Phi$ is a function that selects for every $R \in \mathcal{R}^{N}$ a feasible allocation $\Phi(R) \in X$. Each agent $i \in N$ only cares about his own allotment $\Phi_{i}(R) \in K$.

We extend the original analysis of deterministic rules by considering "probabilistic"

rules. A probabilistic (allocation) rule $\varphi$ is a function that selects for every $R \in \mathcal{R}^{N}$ a (probability) distribution over the set of feasible allocations $X$, denoted by $\varphi(R)$. Given 
$X^{\prime} \subseteq X$, we denote by $\varphi(R)\left(X^{\prime}\right)$ the probability that the distribution $\varphi(R)$ places on the set $X^{\prime}$. Since the set of feasible allocations $X$ is finite, a distribution over $X$ can be interpreted as a lottery, or a simple gamble, on $X$. For $X=\left\{x^{1}, \ldots, x^{|X|}\right\}$ we denote such a distribution over the set of feasible allocations $X$ by $\left[p^{1} \circ x^{1}, \ldots, p^{|X|} \circ x^{|X|}\right]$ where for all $l \in\{1, \ldots,|X|\}$, $p^{l} \in[0,1]$ and $\sum_{l=1}^{|X|} p^{l}=1$. For notational convenience, when formalizing distributions, we will only denote feasible allocations $x^{l}$ that occur with strictly positive probability $p^{l}>0$, e.g., instead of $\left[\frac{1}{2} \circ x^{1}, \frac{1}{2} \circ x^{2}, 0 \circ x^{3}, \ldots, 0 \circ x^{|X|}\right]$ we write $\left[\frac{1}{2} \circ x^{1}, \frac{1}{2} \circ x^{2}\right]$.

For each agent $i \in N$, let $\varphi_{i}(R)$ denote the marginal distribution induced by $\varphi(R)$ over his allotments in $K$. Each agent $i \in N$ only cares about his marginal distribution $\varphi_{i}(R)$ on $K$. A deterministic rule is a probabilistic rule that selects for every $R \in \mathcal{R}^{N}$ a distribution placing probability 1 on a single allocation in $X$.

The following example demonstrates that two distributions having the same marginal distributions need not be equal.

Example 1 Let $N=\{1,2,3\}, k=9, Q=\left[\frac{1}{3} \circ(3,6,0), \frac{1}{3} \circ(0,3,6), \frac{1}{3} \circ(6,0,3)\right]$, and $Q^{\prime}=\left[\frac{1}{3} \circ(3,0,6), \frac{1}{3} \circ(6,3,0), \frac{1}{3} \circ(0,6,3)\right]$. Then for all $i \in N, Q_{i}=Q_{i}^{\prime}$, but $Q \neq Q^{\prime} . \quad \triangleleft$

Remark 1 Let $Q$ be a distribution on $X$. Then, $\sum_{i=1}^{n} \sum_{x_{i}=0}^{k} Q_{i}\left(x_{i}\right) x_{i}=k$. Equivalently to $\sum_{i=1}^{n} \sum_{x_{i}=0}^{k} Q_{i}\left(x_{i}\right) x_{i}$ we also use the notation $\int_{X} x_{i} d Q_{i}$.

We extend preferences on agents' allotments in $K$ to marginal distributions on $K$. Our extension is based on the concept of weak upper contour sets.

Given $x_{i} \in K$ and $R_{i} \in \mathcal{R}$, the weak upper contour set of $x_{i}$ at $R_{i}$ is defined as $B\left(x_{i}, R_{i}\right) \equiv\left\{y_{i} \in K \mid y_{i} R_{i} x_{i}\right\}$. Given a preference relation $R_{i} \in \mathcal{R}$ and two marginal distributions $Q_{i}, Q_{i}^{\prime}$ on $K$, agent $i$ weakly prefers $Q_{i}$ to $Q_{i}^{\prime}$, if $Q_{i}$ assigns to each weak upper contour set at least the probability that is assigned to this set by $Q_{i}^{\prime}$. For notational convenience we use the same symbols $R_{i}$ and $P_{i}$ to define preferences over marginal distributions.

Ordinal Extension of Preferences: For all $R_{i} \in \mathcal{R}$ and all marginal distributions $Q_{i}, Q_{i}^{\prime}$ on $K, Q_{i} R_{i} Q_{i}^{\prime}$ if and only if

$$
\text { for all } x_{i} \in K, Q_{i}\left(B\left(x_{i}, R_{i}\right)\right) \geq Q_{i}^{\prime}\left(B\left(x_{i}, R_{i}\right)\right) \text {. }
$$

Furthermore, $Q_{i} P_{i} Q_{i}^{\prime}$ if and only if $Q_{i} R_{i} Q_{i}^{\prime}$ and

$$
\text { for some } y_{i} \in K, Q_{i}\left(B\left(y_{i}, R_{i}\right)\right)>Q_{i}^{\prime}\left(B\left(y_{i}, R_{i}\right)\right) \text {. }
$$


Inequality (1) is a first order stochastic dominance condition; in particular it requires that the marginal distributions $Q_{i}$ and $Q_{i}^{\prime}$ are comparable in that respect. Therefore, our extension is not complete on the set of all marginal distributions on $K$.

Our extension of preferences is equivalent to the following. Assume that each agent's preference relation over lotteries can be represented by a utility function in the sense that it can be used to compare two marginal distributions via the expected utilities relative to this representation. Then (1) is equivalent to the fact that the expected utility relative to any utility function representing $R_{i}$ is at $Q$ greater or equal than at $Q^{\prime}$. Thus, regardless which utility function represents an agent's preference relation, he will weakly prefer $Q$ to $Q^{\prime}$. For a further discussion of utility representation of preferences we refer to Section 5 .

We are interested in Pareto efficiency. Following the definition of Pareto efficiency for deterministic rules, a probabilistic rule is Pareto efficient if it only assigns "Pareto efficient distributions on $X "$; i.e., a distribution assigned by the probabilistic rule cannot be changed in such a way that no agent is worse off and some agent is better off.

Let $Q, Q^{\prime}$ be distributions on $X$. If for all $i \in N, Q_{i} R_{i} Q_{i}^{\prime}$ and for some $j \in N, Q_{j} P_{j} Q_{j}^{\prime}$, then we call $Q$ a Pareto improvement over $Q^{\prime}$.

Pareto Efficiency: For all $R \in \mathcal{R}^{N}$, there exists no Pareto improvement over $\varphi(R)$.

Remark 2 (Same-Sidedness) A deterministic rule $\Phi$ satisfies Pareto efficiency if and only if $\Phi$ satisfies same-sidedness; i.e., for all $R \in R^{N}$,

(i) if $\sum_{i \in N} p\left(R_{i}\right) \geq k$, then for all $i \in N, \Phi_{i}(R) \leq p\left(R_{i}\right)$ and

(ii) if $\sum_{i \in N} p\left(R_{i}\right) \leq k$, then for all $i \in N, \Phi_{i}(R) \geq p\left(R_{i}\right)$.

A similar result holds for probabilistic rules: ex-post efficiency is equivalent to samesidedness. Given $x_{i}, y_{i} \in K$ such that $x_{i} \leq y_{i}$, let $\left[x_{i}, y_{i}\right] \equiv\left\{x_{i}, x_{i}+1, \ldots, y_{i}\right\}$.

Lemma 1 (Pareto Efficiency) A probabilistic rule $\varphi$ satisfies Pareto efficiency if and only if it satisfies same-sidedness; i.e., for all $R \in \mathcal{R}^{N}$,

(i) if $\sum_{i \in N} p\left(R_{i}\right) \geq k$, then for all $i \in N, \varphi_{i}(R)\left(\left[0, p\left(R_{i}\right)\right]\right)=1$ and

(ii) if $\sum_{i \in N} p\left(R_{i}\right) \leq k$, then for all $i \in N, \varphi_{i}(R)\left(\left[p\left(R_{i}\right), k\right]\right)=1$.

Proof. It is straightforward to show that if $\varphi$ satisfies Pareto efficiency, then (i) and (ii) hold. To show the converse, suppose $\varphi$ satisfies same-sidedness. Suppose that $\varphi$ violates 
Pareto efficiency for some $R \in \mathcal{R}^{N}$. Thus, there exists a distribution $Q$ over $X$ such that for all $i \in N, Q_{i} R_{i} \varphi_{i}(R)$ and for some $j \in N, Q_{j} P_{j} \varphi_{j}(R)$. Without loss of generality, let $k \leq \sum_{i \in N} p\left(R_{i}\right)$ and for all $i \in N, Q_{i}(R)\left(\left[0, p\left(R_{i}\right)\right]\right)=1$. Given $i \in N$, the function $f^{i}: X \rightarrow K$ denotes the projection of $X$ onto $i$ 's coordinate, i.e., for all $x \in X, f^{i}(x)=x_{i}$. Since $Q$ and $\varphi(R)$ satisfy same-sidedness and for all $i \in N, Q_{i} R_{i} \varphi_{i}(R)$, it follows for all $i \in N$,

$$
\int_{X} f^{i} d Q=\int_{\left[0, p\left(R_{i}\right)\right]} x_{i} d Q_{i} \geq \int_{\left[0, p\left(R_{i}\right)\right]} x_{i} d \varphi_{i}(R)=\int_{X} f^{i} d \varphi(R) .
$$

Thus, for all $i \in N, \int_{X} f^{i} d Q \geq \int_{X} f^{i} d \varphi(R)$, and for some $j \in N, \int_{X} f^{j} d Q>\int_{X} f^{j} d \varphi(R)$. Hence,

$$
\int_{X} \sum_{i \in N} f^{i} d Q=\sum_{i \in N} \int_{X} f^{i} d Q>\sum_{i \in N} \int_{X} f^{i} d \varphi(R)=\int_{X} \sum_{i \in N} f^{i} d \varphi(R) .
$$

Note that for all $x \in X, \sum_{i \in N} f^{i}(x)=k$. Hence,

$$
\int_{X} \sum_{i \in N} f^{i} d Q=k \text { and } \int_{X} \sum_{i \in N} f^{i} d \varphi(R)=k .
$$

Now, the previous two facts contradict (3).

Conditions (i) and (ii) in Lemma 1 imply that an allocation chosen by the probabilistic rule $\varphi$ satisfies same-sidedness and therefore Pareto efficiency. Hence, Lemma 1 states that Pareto efficiency and ex-post Pareto efficiency are equivalent in our model. Bogomolnaia and Moulin (1999) show that this equivalence is not valid when assigning heterogenous indivisible objects to individuals.

Next we introduce strategy-proofness for probabilistic rules. By strategy-proofness no agent can ever benefit by misrepresenting his preference relation. ${ }^{3}$

Given $R \in \mathcal{R}^{N}$ and $M \subseteq N$, the restriction $\left(R_{i}\right)_{i \in M} \in \mathcal{R}^{M}$ of $R$ to $M$ is denoted by $R_{M}$. We also use the notation $R_{-i}=R_{N \backslash\{i\}}$. For example, $\left(\bar{R}_{i}, R_{-i}\right)$ denotes the profile obtained from $R$ by replacing $R_{i}$ by $\bar{R}_{i}$.

Strategy-Proofness: $\quad$ For all $R \in \mathcal{R}^{N}$, all $i \in N$, and all $\bar{R}_{i} \in \mathcal{R}, \varphi_{i}(R) R_{i} \varphi_{i}\left(\bar{R}_{i}, R_{-i}\right)$.

\footnotetext{
${ }^{3}$ In game theoretical terms, a rule satisfies strategy-proofness if in its associated direct revelation game form, it is a weakly dominant strategy for each agent to announce his true preference relation.
} 
Note that our notion of strategy-proofness also requires that the marginal distributions that are assigned by the probabilistic rule before and after any unilateral deviation are comparable.

By anonymity the names of the agents do not matter. More precisely, an anonymous probabilistic rule is symmetric in its arguments.

Let $\Pi^{N}$ be the class of all permutations on $N$. Then for all $R \in \mathcal{R}^{N}$ and all $\pi \in \Pi^{N}$, by $R_{\pi}$ we mean $\left(R_{\pi(i)}\right)_{i \in N}$.

Anonymity: For all $R \in \mathcal{R}^{N}$, all $\pi \in \Pi^{N}$, and all $i \in N, \varphi_{\pi(i)}(R)=\varphi_{i}\left(R_{\pi}\right)$.

No-envy states that no agent strictly prefers the marginal distribution of another agent to his own.

No-Envy: $\quad$ For all $R \in \mathcal{R}^{N}$ and all $i, j \in N, \varphi_{i}(R) R_{i} \varphi_{j}(R)$.

No-envy also requires that the agents' marginal distributions that are assigned by the probabilistic rule are comparable.

Equal treatment of equals, a weakening of no-envy and of anonymity, requires that if two agents have the same preference relations, then each of them is indifferent between his marginal distribution and the other agent's marginal distribution.

Equal Treatment of Equals: For all $R \in \mathcal{R}^{N}$ and all $i, j \in N$, if $R_{i}=R_{j}$, then $\varphi_{i}(R) I_{i} \varphi_{j}(R)$.

\section{The Uniform Probabilistic Correspondence}

In identifying probabilistic rules that satisfy a certain combination of properties, we will not be able to determine the exact distribution for each profile. All requirements are formulated with respect to marginal distributions and as demonstrated in Example 1 those do not uniquely determine the original distribution. We will only be able to show that a probabilistic rule satisfies a certain list of requirements if and only if for each profile the marginal distributions are of a certain form. Therefore, we introduce correspondences that assign to each profile a set of distributions. 
The following "uniform correspondence" assigns to each profile exactly the distributions that induce "uniform marginal distributions" on each agent's allotments: for each profile of peaks $\left(p\left(R_{i}\right)\right)_{i \in N}$ calculate the so-called uniform allocation for $R$. Denote this allocation by $\tilde{U}(R)$. Without loss of generality, suppose that $R$ is in excess demand, i.e., $\sum_{i \in N} p\left(R_{i}\right)>k$ (the definition for the excess supply case is similar). Thus, for some $\lambda \in[0, k]$, we have that for all $i \in N, \tilde{U}_{i}(R)=\min \left(p\left(R_{i}\right), \lambda\right)$. For each agent we choose the following uniform marginal distribution $f_{i}(R)$ over his allotments. If $\tilde{U}_{i}(R)=p\left(R_{i}\right)$, then $f_{i}(R)$ places probability 1 on $p\left(R_{i}\right)$. Otherwise, calculate $x_{\lambda} \in\{0,1, \ldots, k\}$ such that $\lambda$ belongs to the interval with endpoints $x_{\lambda}$ and $x_{\lambda}+1$. Then calculate weights $\alpha$ and $(1-\alpha)$ on the endpoints such that $\lambda$ equals the convex combination of the two endpoints according to the weights, i.e., $\lambda=\alpha x_{\lambda}+(1-\alpha)\left(x_{\lambda}+1\right)$ where $\alpha \in[0,1]$. Then, the uniform marginal distribution $f_{i}(R)$ places probability $\alpha$ on $x_{\lambda}$ and probability $1-\alpha$ on $x_{\lambda}+1$.

Uniform Probabilistic Correspondence, $\mathcal{U}$ : Let $R \in \mathcal{R}^{N}$ and $Q$ be a distribution over $X$. Then $Q \in \mathcal{U}(R)$ if and only if the following holds.

(i) Excess Demand: $\sum_{i \in N} p\left(R_{i}\right)>k$.

Let $\tilde{U}_{i}(R)=\min \left(p\left(R_{i}\right), \lambda\right)$ where $\lambda \in \mathbb{R}_{+}$solves $\sum_{i \in N} \tilde{U}_{i}(R)=k$.

Determine $x_{\lambda} \in K$ such that $\lambda \in\left[x_{\lambda}, x_{\lambda}+1[\right.$. Then for all $i \in N$,

(a) if $p\left(R_{i}\right) \leq x_{\lambda}$, then $Q_{i}\left(p\left(R_{i}\right)\right)=1$ and

(b) if $p\left(R_{i}\right) \geq x_{\lambda}+1$, then $Q_{i}\left(x_{\lambda}+1\right)=\lambda-x_{\lambda}$ and $Q_{i}\left(x_{\lambda}\right)=1-\left(\lambda-x_{\lambda}\right)$.

(ii) Balanced Demand: $\sum_{i \in N} p\left(R_{i}\right)=k$. Then for all $i \in N, Q_{i}\left(p\left(R_{i}\right)\right)=1$.

(iii) Excess Supply: $\sum_{i \in N} p\left(R_{i}\right)<k$.

Let $\tilde{U}_{i}(R)=\max \left(p\left(R_{i}\right), \lambda\right)$ where $\lambda \in \mathbb{R}_{+}$solves $\sum_{i \in N} \tilde{U}_{i}(R)=k$.

Determine $x_{\lambda} \in K$ such that $\left.\left.\lambda \in\right] x_{\lambda}, x_{\lambda}+1\right]$. Then for all $i \in N$,

(a) if $p\left(R_{i}\right) \geq x_{\lambda}+1$, then $Q_{i}\left(p\left(R_{i}\right)\right)=1$ and

(b) if $p\left(R_{i}\right) \leq x_{\lambda}$, then $Q_{i}\left(x_{\lambda}+1\right)=\lambda-x_{\lambda}$ and $Q_{i}\left(x_{\lambda}\right)=1-\left(\lambda-x_{\lambda}\right)$.

We say that a probabilistic rule $\varphi$ is a uniform probabilistic rule if for all $R \in \mathcal{R}^{N}, \varphi(R) \in$ $\mathcal{U}(R)$. The following example of a probabilistic rule proves that the uniform correspondence is non-empty. 
Example 2 We define the uniform probabilistic rule $U$ as follows (Sasaki, 1997; in defining the uniform probabilistic rule $U$ Moulin (2000) uses the same trick as we do ${ }^{4}$ in defining the correspondence $\mathcal{U}$ ). Let $R \in \mathcal{R}^{N}$.

(i) Excess Demand: $\sum_{i \in N} p\left(R_{i}\right)>k$.

Without loss of generality, let $\bar{N}=\left\{i \in N \mid p\left(R_{i}\right) \geq x_{\lambda}+1\right\}=\{1, \ldots, \bar{n}\}$ and $\tilde{N}=\left\{i \in N \mid p\left(R_{i}\right) \leq x_{\lambda}\right\}=\{\bar{n}+1, \ldots, n\}$. Then we obtain $U(R)$ as follows: in each allocation that occurs at $U(R)$ with positive probability, each agent in $\tilde{N}$ receives his peak amount and each agent in $\bar{N}$ receives either $x_{\lambda}$ or $x_{\lambda}+1$. Note that for each $i \in \bar{N},\left(x_{\lambda}+1\right) P_{i} x_{\lambda}$ and that exactly $\bar{n}\left(\lambda-x_{\lambda}\right)$ agents in $\bar{N}$ can receive $x_{\lambda}+1$. We obtain $U(R)$ by placing equal probability on all allocations where all agents in $\tilde{N}$ receive their peak amounts, $\bar{n}\left(\lambda-x_{\lambda}\right)$ agents in $\bar{N}$ receive $x_{\lambda}+1$, and the remaining agents in $\bar{N}$ receive $x_{\lambda}$. Hence, $U(R)$ is obtained by placing equal probabilities on exactly $\left(\begin{array}{c}\bar{n} \\ \bar{n}\left(\lambda-x_{\lambda}\right)\end{array}\right)$ allocations. Note that

(a) if $p\left(R_{i}\right) \leq x_{\lambda}$, then $U_{i}(R)\left(p\left(R_{i}\right)\right)=1$ and

(b) if $p\left(R_{i}\right) \geq x_{\lambda}+1$, then $U_{i}(R)\left(x_{\lambda}+1\right)=\lambda-x_{\lambda}$ and $U_{i}(R)\left(x_{\lambda}\right)=1-\left(\lambda-x_{\lambda}\right)$.

(ii) Balanced Demand: $\sum_{i \in N} p\left(R_{i}\right)=k$. Then, $U(R)=\left[1 \circ\left(p\left(R_{1}\right), \ldots, p\left(R_{n}\right)\right)\right]$.

(iii) Excess Supply: $\sum_{i \in N} p\left(R_{i}\right)<k$.

Without loss of generality, let $\bar{N}=\left\{i \in N \mid p\left(R_{i}\right) \leq x_{\lambda}\right\}=\{1, \ldots, \bar{n}\}$ and $\tilde{N}=\{i \in$ $\left.N \mid p\left(R_{i}\right) \geq x_{\lambda}+1\right\}=\{\bar{n}+1, \ldots, n\}$. Then we obtain $U(R)$ as follows: in each allocation that occurs at $U(R)$ with positive probability, each agent in $\tilde{N}$ receives his peak amount and each agent in $\bar{N}$ receives either $x_{\lambda}$ or $x_{\lambda}+1$. Note that for $i \in \bar{N}$, $x_{\lambda} P_{i}\left(x_{\lambda}+1\right)$ and that exactly $\bar{n}-\bar{n}\left(\lambda-x_{\lambda}\right)$ agents in $\bar{N}$ can receive $x_{\lambda}$. In other words, $\bar{n}\left(\lambda-x_{\lambda}\right)$ of agents in $\bar{N}$ will receive $x_{\lambda}+1$. We obtain $U(R)$ by placing equal probability on all allocations where all agents in $\tilde{N}$ receive their peak amounts, $\bar{n}\left(\lambda-x_{\lambda}\right)$ agents in $\bar{N}$ receive $x_{\lambda}+1$, and the remaining agents in $\bar{N}$ receive $x_{\lambda}$. Hence, $U(R)$ is obtained by placing equal probabilities on exactly $\left(\begin{array}{c}\bar{n} \\ \bar{n}\left(\lambda-x_{\lambda}\right)\end{array}\right)$ allocations. Note that

(a) if $p\left(R_{i}\right) \geq x_{\lambda}+1$, then $U_{i}(R)\left(p\left(R_{i}\right)\right)=1$ and

(b) if $p\left(R_{i}\right) \leq x_{\lambda}$, then $U_{i}(R)\left(x_{\lambda}+1\right)=\lambda-x_{\lambda}$ and $U_{i}(R)\left(x_{\lambda}\right)=1-\left(\lambda-x_{\lambda}\right)$.

\footnotetext{
${ }^{4}$ Moulin (2000) and we found this trick independently.
} 
Example 3 Let $N=\{1,2,3,4\}$ and $k=2$. Let $R \in R^{N}$ be such that for all $i \in N$, $p\left(R_{i}\right)=1$. Then, $\bar{N}=N$ and

$$
\begin{gathered}
U(R)=\left[\frac{1}{6} \circ(1,1,0,0), \frac{1}{6} \circ(1,0,1,0), \frac{1}{6} \circ(1,0,0,1),\right. \\
\left.\frac{1}{6} \circ(0,1,1,0), \frac{1}{6} \circ(0,1,0,1), \frac{1}{6} \circ(0,0,1,1)\right] .
\end{gathered}
$$

Let $Q=\left[\frac{1}{2} \circ(1,1,0,0), \frac{1}{2} \circ(0,0,1,1)\right]$ and $Q^{\prime}=\left[\frac{1}{2} \circ(1,0,0,1), \frac{1}{2} \circ(0,1,1,0)\right]$. Then for all $i \in N, Q_{i}=U_{i}(R)=Q_{i}^{\prime}\left(Q, Q^{\prime}, U(R) \in \mathcal{U}(R)\right)$, but $U(R) \neq Q \neq Q^{\prime} \neq U(R)$.

Our main result is that, similarly as in the deterministic setting (Sprumont, 1991; Ching, 1992; Ehlers, 2000), Pareto efficiency, strategy-proofness, and no-envy determine "uniform allocations".

Theorem 1 Uniform probabilistic rules are the only probabilistic rules satisfying Pareto efficiency, strategy-proofness, and no-envy.

Remark 3 Theorem 1 is a tight characterization since we already know from the deterministic framework that all properties are logically independent (see Sprumont 1991 and Ching 1992). However, in contrast to the deterministic framework, we cannot replace no-envy with anonymity or weaken it to equal treatment of equals (see Example 4).

Example 4 Let $N=\{1,2\}$ and $R \in R^{N}$.

- If $p\left(R_{1}\right)+p\left(R_{2}\right) \neq k$, then

$$
\psi(R)=\left[\frac{1}{2} \circ\left(p\left(R_{1}\right), k-p\left(R_{1}\right)\right), \frac{1}{2} \circ\left(k-p\left(R_{2}\right), p\left(R_{2}\right)\right)\right] .
$$

- If $p\left(R_{1}\right)+p\left(R_{2}\right)=k$, then

$$
\psi(R)=\left[1 \circ\left(p\left(R_{1}\right), p\left(R_{2}\right)\right)\right]
$$

The probabilistic rule $\psi$ satisfies Pareto efficiency, strategy-proofness, and anonymity. However, as we will demonstrate next, $\psi$ violates no-envy.

Let $k=5, p\left(R_{1}\right)=5, p\left(R_{2}\right)=1$ and $5 P_{2} 0$. Then,

$$
\psi(R)=\left[\frac{1}{2} \circ(5,0), \frac{1}{2} \circ(4,1)\right]
$$

and

$$
\psi_{2}(R)\left(B\left(5, R_{2}\right)\right)=\frac{1}{2}<1=\psi_{1}(R)\left(B\left(5, R_{2}\right)\right) .
$$

Thus, we do not have that $\psi_{2}(R) R_{2} \psi_{1}(R)$, which contradicts no-envy; more precisely, the comparability condition that is incorporated in no-envy is violated. 
Example 4 can be easily extended to an arbitrary number of agents. More precisely, the rule $\psi$ is the random dictatorship rule for two agents. For an arbitrary number of agents, the random dictatorship rule is Pareto efficient, strategy-proof, and anonymous. Note that all convex combinations of uniform probabilistic rules and the random dictatorship rule satisfy Pareto efficiency, strategy-proofness, and anonymity. It is an open question whether there are other probabilistic rules that satisfy this list of properties.

\section{Proof of the Characterization}

It is straightforward to check that each uniform probabilistic rule satisfies Pareto efficiency, strategy-proofness, and no-envy. Conversely, let $\varphi$ be a probabilistic rule satisfying Pareto efficiency, strategy-proofness, and no-envy. We have to show that for all $R \in \mathcal{R}^{N}, \varphi(R) \in$ $\mathcal{U}(R)$.

Pareto efficiency and Lemma 1 imply that if demand is balanced, then (ii) in the definition of $\mathcal{U}$ holds. In the remainder of this section we prove that if $R$ is in excess demand, then (i) in the definition of $\mathcal{U}$ holds. The proof of (iii), i.e., excess supply, is similar.

Recall that for all $x_{i}, y_{i} \in K$ such that $x_{i} \leq y_{i}$, we have $\left[x_{i}, y_{i}\right] \equiv\left\{x_{i}, x_{i}+1, \ldots, y_{i}\right\}$.

Lemma 2 Let $R \in \mathcal{R}^{N}$ be such that $\sum_{i \in N} p\left(R_{i}\right)>k$. Then for all $i \in N$,

$$
\varphi_{i}(R)\left(\left[\min \left(p\left(R_{i}\right), x_{\lambda}\right), p\left(R_{i}\right)\right]\right)=1 .
$$

Proof. Let $i \in N$. We consider two cases.

Case 1: $p\left(R_{i}\right) \geq x_{\lambda}+1$. We have to show that $\varphi_{i}(R)\left(\left[x_{\lambda}, p\left(R_{i}\right)\right]\right)=1$. Assume, by contradiction, that

$$
\varphi_{i}(R)\left(\left[x_{\lambda}, p\left(R_{i}\right)\right]\right)<1
$$

Let $R_{i}^{\prime} \in \mathcal{R}$ be such that $p\left(R_{i}^{\prime}\right)=p\left(R_{i}\right)$ and for all $x_{i} \in\left[p\left(R_{i}\right), k\right], B\left(x_{i}, R_{i}^{\prime}\right)=\left[p\left(R_{i}\right), x_{i}\right]$. Define $R^{\prime} \equiv\left(R_{i}^{\prime}, R_{-i}\right)$. By (4), Pareto efficiency (PE), and strategy-proofness (SP),

$$
\begin{array}{rll}
1 & \stackrel{(4)}{>} & \varphi_{i}(R)\left(\left[x_{\lambda}, p\left(R_{i}\right)\right]\right) \\
& \stackrel{P E}{=} & \varphi_{i}(R)\left(B\left(x_{\lambda}, R_{i}\right)\right) \\
& \stackrel{S P}{\geq} & \varphi_{i}\left(R^{\prime}\right)\left(B\left(x_{\lambda}, R_{i}\right)\right) \\
& \stackrel{P E}{=} & \varphi_{i}\left(R^{\prime}\right)\left(\left[x_{\lambda}, p\left(R_{i}\right)\right]\right) .
\end{array}
$$


Thus,

$$
\varphi_{i}\left(R^{\prime}\right)\left(\left[x_{\lambda}, p\left(R_{i}\right)\right]\right)<1 .
$$

Hence, by Pareto efficiency (PE), no-envy (NE), and the construction of $R_{i}^{\prime}$, for all $j \neq i$,

$$
\begin{aligned}
& 1 \stackrel{(5)}{>} \varphi_{i}\left(R^{\prime}\right)\left(\left[x_{\lambda}, p\left(R_{i}\right)\right]\right) \\
& \stackrel{P E}{=} \varphi_{i}\left(R^{\prime}\right)\left(\left[x_{\lambda}, k\right]\right)=\varphi_{i}\left(R^{\prime}\right)\left(B\left(x_{\lambda}, R_{i}^{\prime}\right)\right) \\
& \stackrel{N E}{\geq} \varphi_{j}\left(R^{\prime}\right)\left(B\left(x_{\lambda}, R_{i}^{\prime}\right)\right)=\varphi_{j}\left(R^{\prime}\right)\left(\left[x_{\lambda}, k\right]\right) .
\end{aligned}
$$

Thus, for all $j \in N$,

$$
\varphi_{j}\left(R^{\prime}\right)\left(\left[x_{\lambda}, k\right]\right)<1
$$

Let $S_{\lambda} \equiv\left\{j \in N \mid p\left(R_{j}\right) \geq x_{\lambda}+1\right\}$. By the definition of $\lambda$ and $\sum_{j \in N} p\left(R_{j}^{\prime}\right)=\sum_{j \in N} p\left(R_{j}\right)>$ $k, S_{\lambda} \neq \emptyset$.

For all $T \subseteq S_{\lambda}$, define $R^{T}=\left(\left(R_{j}^{T}\right)_{j \in T}, R_{N \backslash T}^{\prime}\right)$ as follows. For all $j \in T$,

- $p\left(R_{j}^{T}\right)=x_{\lambda}$ and

- for all $x_{j} \in\left[x_{\lambda}, k\right], B\left(x_{j}, R_{j}^{T}\right)=\left[x_{\lambda}, x_{j}\right]$.

Let $j \in S_{\lambda}$. If $\sum_{l \in N} p\left(R_{l}^{\{j\}}\right)>k$, then by (6) and strategy-proofness (SP),

$$
\begin{aligned}
1 & \stackrel{(6)}{>} \varphi_{j}\left(R^{\prime}\right)\left(\left[x_{\lambda}, k\right]\right) \geq \varphi_{j}\left(R^{\prime}\right)\left(B\left(x_{\lambda}, R_{j}^{\prime}\right)\right) \\
& \stackrel{S P}{\geq} \varphi_{j}\left(R^{\{j\}}\right)\left(B\left(x_{\lambda}, R_{j}^{\prime}\right)\right) \geq \varphi_{j}\left(R^{\{j\}}\right)\left(x_{\lambda}\right) \\
& =\varphi_{j}\left(R^{\{j\}}\right)\left(p\left(R_{j}^{\{j\}}\right)\right)=\varphi_{j}\left(R^{\{j\}}\right)\left(\left[x_{\lambda}, k\right]\right) .
\end{aligned}
$$

By (7) and no-envy (NE), for all $l \neq j$,

$$
\begin{aligned}
1 & \stackrel{(7)}{>} \varphi_{j}\left(R^{\{j\}}\right)\left(\left[x_{\lambda}, k\right]\right)=\varphi_{j}\left(R^{\{j\}}\right)\left(B\left(k, R_{j}^{\{j\}}\right)\right) \\
& \stackrel{N E}{\geq} \varphi_{l}\left(R^{\{j\}}\right)\left(B\left(k, R_{j}^{\{j\}}\right)\right)=\varphi_{l}\left(R^{\{j\}}\right)\left(\left[x_{\lambda}, k\right]\right) .
\end{aligned}
$$

Thus, for all $l \in N, \varphi_{l}\left(R^{\{j\}}\right)\left(\left[x_{\lambda}, k\right]\right)<1$. Hence, by repeated application of the above arguments, if for $T \subseteq S_{\lambda}, \sum_{l \in N} p\left(R_{l}^{T}\right)>k$, then for all $l \in N$,

$$
\varphi_{l}\left(R^{T}\right)\left(\left[x_{\lambda}, k\right]\right)<1
$$

Now fix $T_{0} \subseteq S_{\lambda}$ such that for some $j \in S_{\lambda} \backslash T_{0}, \sum_{l \in N} p\left(R_{l}^{T_{0} \cup\{j\}}\right) \leq k<\sum_{l \in N} p\left(R_{l}^{T_{0}}\right)$. 
By the definition of $\lambda, T_{0} \neq \emptyset$. Let $\bar{R}_{j} \in \mathcal{R}$ be such that $p\left(\bar{R}_{j}\right)=k-\sum_{l \neq j} p\left(R_{l}^{T_{0}}\right)$. Define $\bar{R} \equiv\left(\bar{R}_{j}, R_{-j}^{T_{0}}\right)$. Note that

$$
x_{\lambda} \leq p\left(\bar{R}_{j}\right)<p\left(R_{j}^{T_{0}}\right) \text { and } \sum_{l \in N} p\left(\bar{R}_{l}\right)=k
$$

By Pareto efficiency, $\varphi_{j}(\bar{R})\left(p\left(\bar{R}_{j}\right)\right)=1$. Since $p\left(\bar{R}_{j}\right) \in B\left(x_{\lambda}, R_{j}^{T_{0}}\right)$, it follows that $\varphi_{j}(\bar{R})\left(B\left(x_{\lambda}, R_{j}^{T_{0}}\right)\right)=$ 1. But this implies a contradiction because by strategy-proofness (SP),

$$
\begin{aligned}
1 & \stackrel{(8)}{>} \varphi_{j}\left(R^{T_{0}}\right)\left(\left[x_{\lambda}, k\right]\right) \geq \varphi_{j}\left(R^{T_{0}}\right)\left(B\left(x_{\lambda}, R_{j}^{T_{0}}\right)\right) \\
& \quad \geq \varphi_{j}(\bar{R})\left(B\left(x_{\lambda}, R_{j}^{T_{0}}\right)\right)=1 .
\end{aligned}
$$

Therefore, (4) was wrong and the statement for Case 1 is proven.

Case 2: $p\left(R_{i}\right) \leq x_{\lambda}$. We have to show that $\varphi_{i}(R)\left(p\left(R_{i}\right)\right)=1$. Assume, by contradiction, that

$$
\varphi_{i}(R)\left(p\left(R_{i}\right)\right)<1
$$

Let $R_{i}^{\prime} \in \mathcal{R}$ be such that $p\left(R_{i}^{\prime}\right)=p\left(R_{i}\right)$ and for all $x_{i} \in\left[p\left(R_{i}\right), k\right], B\left(x_{i}, R_{i}^{\prime}\right)=\left[p\left(R_{i}\right), x_{i}\right]$. Define $R^{\prime} \equiv\left(R_{i}^{\prime}, R_{-i}\right)$. By strategy-proofness and $(9), \varphi_{i}\left(R^{\prime}\right)\left(p\left(R_{i}\right)\right)<1$. By Pareto efficiency (PE), no-envy (NE), and the construction of $R_{i}^{\prime}$, for all $j \in N$,

$$
\begin{array}{rll}
1 & > & \varphi_{i}\left(R^{\prime}\right)\left(p\left(R_{i}\right)\right) \\
& \stackrel{P E}{=} \varphi_{i}\left(R^{\prime}\right)\left(\left[p\left(R_{i}\right), k\right]\right)=\varphi_{i}\left(R^{\prime}\right)\left(B\left(k, R_{i}^{\prime}\right)\right) \\
& \stackrel{N E}{\geq} \varphi_{j}\left(R^{\prime}\right)\left(B\left(k, R_{i}^{\prime}\right)\right)=\varphi_{j}\left(R^{\prime}\right)\left(\left[p\left(R_{i}\right), k\right]\right) .
\end{array}
$$

Particularly, because $p\left(R_{i}\right) \leq x_{\lambda}$, for all $j \in N$,

$$
\varphi_{j}\left(R^{\prime}\right)\left(\left[x_{\lambda}, k\right]\right)<1
$$

However, for some $j \in N, p\left(R_{j}^{\prime}\right) \geq x_{\lambda}+1$. Hence, in contradiction to (10), by Case 1 and Pareto efficiency $(P E), 1=\varphi_{j}\left(R^{\prime}\right)\left(\left[x_{\lambda}, p\left(R_{j}^{\prime}\right)\right]\right) \stackrel{P E}{=} \varphi_{j}\left(R^{\prime}\right)\left(\left[x_{\lambda}, k\right]\right)$.

To complete the proof of Theorem 1, we prove that for all $R \in \mathcal{R}^{N}$, if $\sum_{i \in N} p\left(R_{i}\right)>k$, then for all $i \in N, \varphi_{i}(R)=U_{i}(R)$; i.e.,

(a) if $p\left(R_{i}\right) \leq x_{\lambda}$, then $\varphi_{i}(R)\left(p\left(R_{i}\right)\right)=1=U_{i}(R)\left(p\left(R_{i}\right)\right)$ and

(b) if $p\left(R_{i}\right) \geq x_{\lambda}+1$, then $\varphi_{i}(R)\left(x_{\lambda}+1\right)=\lambda-x_{\lambda}=U_{i}(R)\left(x_{\lambda}+1\right)$ and $\varphi_{i}(R)\left(x_{\lambda}\right)=$ $1-\left(\lambda-x_{\lambda}\right)=U_{i}(R)\left(x_{\lambda}\right)$. 
Let $R \in \mathcal{R}^{N}$ be such that $\sum_{i \in N} p\left(R_{i}\right)>k$. By Lemma 2 , for all $i \in N, \varphi_{i}(R)\left(\left[\min \left(p\left(R_{i}\right), x_{\lambda}\right), p\left(R_{i}\right)\right]\right)=$ 1. Thus, for all $i \in N$, if $p\left(R_{i}\right) \leq x_{\lambda}$, then

$$
\varphi_{i}(R)\left(p\left(R_{i}\right)\right)=1
$$

Hence, (11) implies (a); i.e., if $p\left(R_{i}\right) \leq x_{\lambda}$, then $\varphi_{i}(R)=U_{i}(R)$.

Next, we prove (b); i.e., if $p\left(R_{i}\right) \geq x_{\lambda}+1$, then $\varphi_{i}(R)=U_{i}(R)$. We consider two cases.

Case 1: For some $j \in N, R_{j} \in \mathcal{R}$ is such that $p\left(R_{j}\right)=x_{\lambda}+1$, and for all $x_{j} \in\left[p\left(R_{j}\right), k\right]$, $B\left(x_{j}, R_{j}\right)=\left[p\left(R_{j}\right), x_{j}\right]$.

By Pareto efficiency (PE), no-envy (NE), and the structure of $R_{j}$, for all $i \in N$ such that $p\left(R_{i}\right) \geq x_{\lambda}+1$,

$$
\begin{aligned}
\varphi_{j}(R)\left(p\left(R_{j}\right)\right) & \stackrel{P E}{=} \varphi_{j}(R)\left(B\left(k, R_{j}\right)\right) \\
& \stackrel{N E}{\geq} \varphi_{i}(R)\left(B\left(k, R_{j}\right)\right) \\
& \stackrel{P E}{=} \varphi_{i}(R)\left(\left[x_{\lambda}+1, p\left(R_{i}\right)\right]\right) .
\end{aligned}
$$

Similarly, for all $i \in N$ such that $p\left(R_{i}\right) \geq x_{\lambda}+1$,

$$
\begin{aligned}
\varphi_{i}(R)\left(\left[x_{\lambda}+1, p\left(R_{i}\right)\right]\right) & \stackrel{P E}{=} \varphi_{i}(R)\left(B\left(x_{\lambda}+1, R_{i}\right)\right) \\
& \stackrel{N E}{\geq} \varphi_{j}(R)\left(B\left(x_{\lambda}+1, R_{i}\right)\right) \\
& \stackrel{P E}{=} \varphi_{j}(R)\left(p\left(R_{j}\right)\right) .
\end{aligned}
$$

Hence, for all $i \in N$ such that $p\left(R_{i}\right) \geq x_{\lambda}+1$,

$$
\varphi_{i}(R)\left(\left[x_{\lambda}+1, p\left(R_{i}\right)\right]\right)=\varphi_{j}(R)\left(p\left(R_{j}\right)\right) .
$$

Thus, by Lemma 2 , for all $i \in N$ such that $p\left(R_{i}\right) \geq x_{\lambda}+1$,

$$
\varphi_{i}(R)\left(x_{\lambda}\right)=\varphi_{j}(R)\left(x_{\lambda}\right)=1-\varphi_{j}(R)\left(p\left(R_{j}\right)\right) .
$$

We consider two subcases.

Case 1.1: For all $i \in N, p\left(R_{i}\right) \leq x_{\lambda}+1$.

Thus, by Pareto efficiency, for all $i \in N, \varphi_{i}(R)\left(\left[x_{\lambda}+2, k\right]\right)=0$. Then by Lemma 2 and (13), for all $i, l \in N$ such that $p\left(R_{i}\right)=p\left(R_{l}\right)=x_{\lambda}+1$,

$$
\varphi_{i}(R)\left(x_{\lambda}\right)=\varphi_{l}(R)\left(x_{\lambda}\right) \text { and } \varphi_{i}(R)\left(x_{\lambda}+1\right)=\varphi_{l}(R)\left(x_{\lambda}+1\right) .
$$


Recall that by Remark 1 and (a),

$$
\sum_{\substack{i \in N \text { such that } \\ p\left(R_{i}\right)=x_{\lambda}+1}} \varphi_{i}(R)\left(x_{\lambda}\right) x_{\lambda}+\sum_{\substack{i \in N \text { such that } \\ p\left(R_{i}\right)=x_{\lambda}+1}} \varphi_{i}(R)\left(x_{\lambda}+1\right)\left(x_{\lambda}+1\right)=k-\sum_{\substack{i \in N \text { such that } \\ p\left(R_{i}\right) \leq x_{\lambda}}} p\left(R_{i}\right) .
$$

Recall that by the definition of $\lambda$ and $x_{\lambda}$,

$$
k=\sum_{i \in N} \min \left(p\left(R_{i}\right), \lambda\right)=\sum_{\substack{i \in N \text { such that } \\ p\left(R_{i}\right) \leq x_{\lambda}}} p\left(R_{i}\right)+\sum_{\substack{i \in N \text { such that } \\ p\left(R_{i}\right)=x_{\lambda}+1}} \lambda .
$$

Furthermore, note that for all $i \in N$ such that $p\left(R_{i}\right)=x_{\lambda}+1$,

$$
\varphi_{i}(R)\left(x_{\lambda}\right)+\varphi_{i}(R)\left(x_{\lambda}+1\right)=1 .
$$

Using (15), (16), and (17), it follows that

$$
\sum_{\substack{i \in N \text { such that } \\ p\left(R_{i}\right)=x_{\lambda}+1}} x_{\lambda}+\sum_{\substack{i \in N \text { such that } \\ p\left(R_{i}\right)=x_{\lambda}+1}} \varphi_{i}(R)\left(x_{\lambda}+1\right)=\sum_{\substack{i \in N \text { such that } \\ p\left(R_{i}\right)=x_{\lambda}+1}} \lambda .
$$

This and (14) imply that for all $i \in N$ such that $p\left(R_{i}\right)=x_{\lambda}+1, \varphi_{i}(R)\left(x_{\lambda}+1\right)=\lambda-x_{\lambda}$. Thus, for all $i \in N$ such that $p\left(R_{i}\right)=x_{\lambda}+1, \varphi_{i}(R)\left(x_{\lambda}\right)=1-\left(\lambda-x_{\lambda}\right)$. This proves (b); i.e., if $p\left(R_{i}\right) \geq x_{\lambda}+1$, then $\varphi_{i}(R)=U_{i}(R)$. This completes the proof for Case 1.1.

Case 1.2: For some $l \in N, p\left(R_{l}\right) \geq x_{\lambda}+2$.

If for all $l \in N$ such that $p\left(R_{l}\right) \geq x_{\lambda}+2, \varphi_{l}(R)\left(\left[x_{\lambda}+2, k\right]\right)=0$, then similarly as in Case 1.1 it follows that $p\left(R_{l}\right) \geq x_{\lambda}+1$ implies $\varphi_{l}(R)=U_{l}(R)$. Assume, by contradiction, that for some $l \in N$ such that $p\left(R_{l}\right) \geq x_{\lambda}+2$,

$$
\varphi_{l}(R)\left(\left[x_{\lambda}+2, k\right]\right)>0 .
$$

Let $R_{l}^{\prime} \in \mathcal{R}$ be such that $R_{l}^{\prime}=R_{j}$ and $R^{\prime} \equiv\left(R_{l}^{\prime}, R_{-l}\right)$. By Pareto efficiency (PE) and strategy-proofness (SP),

$$
\begin{aligned}
\varphi_{l}\left(R^{\prime}\right)\left(x_{\lambda}+1\right) & \stackrel{P E}{=} \varphi_{l}\left(R^{\prime}\right)\left(\left[x_{\lambda}+1, k\right]\right)=\varphi_{l}\left(R^{\prime}\right)\left(B\left(k, R_{l}^{\prime}\right)\right) \\
& \stackrel{S P}{\geq} \varphi_{l}(R)\left(B\left(k, R_{l}^{\prime}\right)\right)=\varphi_{l}(R)\left(\left[x_{\lambda}+1, k\right]\right)
\end{aligned}
$$

and

$$
\begin{aligned}
\varphi_{l}(R)\left(\left[x_{\lambda}+1, k\right]\right) & \stackrel{P E}{=} \varphi_{l}(R)\left(B\left(x_{\lambda}+1, R_{l}\right)\right) \\
& \stackrel{S P}{\geq} \varphi_{l}\left(R^{\prime}\right)\left(B\left(x_{\lambda}+1, R_{l}\right)\right) \\
& \stackrel{P E}{=} \varphi_{l}\left(R^{\prime}\right)\left(x_{\lambda}+1\right) .
\end{aligned}
$$


Hence,

$$
\begin{aligned}
\varphi_{l}\left(R^{\prime}\right)\left(x_{\lambda}+1\right) & =\varphi_{l}(R)\left(\left[x_{\lambda}+1, k\right]\right) \text { and } \\
\varphi_{l}\left(R^{\prime}\right)\left(x_{\lambda}\right) & =\varphi_{l}(R)\left(x_{\lambda}\right) .
\end{aligned}
$$

Suppose that for all $i \in N$ such that $p\left(R_{i}^{\prime}\right) \geq x_{\lambda}+2, \varphi_{i}\left(R^{\prime}\right)\left(\left[x_{\lambda}+2, k\right]\right)=0$. Let $N^{\prime} \equiv\{i \in$ $\left.N \mid p\left(R_{i}\right) \geq x_{\lambda}+1\right\}=\left\{i \in N \mid p\left(R_{i}^{\prime}\right) \geq x_{\lambda}+1\right\}$.

Consider profile $R$ and recall from Case 1 that for all $i \in N^{\prime}, \varphi_{i}(R)\left(\left[x_{\lambda}+1, p\left(R_{i}\right)\right]\right)=$ $\varphi_{j}(R)\left(p\left(R_{j}\right)\right)$ and $\varphi_{i}(R)\left(x_{\lambda}\right)=\varphi_{j}(R)\left(x_{\lambda}\right)$. In particul, for all $i \in N^{\prime}$,

$$
\begin{gathered}
\varphi_{i}(R)\left(\left[x_{\lambda}+1, p\left(R_{i}\right)\right]\right)=\varphi_{j}(R)\left(p\left(R_{j}\right)\right)=\varphi_{l}(R)\left(\left[x_{\lambda}+1, p\left(R_{l}\right)\right]\right) \text { and } \\
\varphi_{i}(R)\left(x_{\lambda}\right)=\varphi_{j}(R)\left(x_{\lambda}\right)=\varphi_{l}(R)\left(x_{\lambda}\right) .
\end{gathered}
$$

Consider profile $R^{\prime}$ and recall from Case 1 that for all $i \in N^{\prime}, \varphi_{i}\left(R^{\prime}\right)\left(\left[x_{\lambda}+1, p\left(R_{i}^{\prime}\right)\right]\right)=$ $\varphi_{j}\left(R^{\prime}\right)\left(p\left(R_{j}\right)\right)$ and $\varphi_{i}\left(R^{\prime}\right)\left(x_{\lambda}\right)=\varphi_{j}\left(R^{\prime}\right)\left(x_{\lambda}\right)$. In particular, for all $i \in N^{\prime}$,

$$
\begin{aligned}
\varphi_{i}\left(R^{\prime}\right)\left(x_{\lambda}+1\right) & =\varphi_{j}\left(R^{\prime}\right)\left(p\left(R_{j}^{\prime}\right)\right)=\varphi_{l}\left(R^{\prime}\right)\left(x_{\lambda}+1\right) \text { and } \\
\varphi_{i}\left(R^{\prime}\right)\left(x_{\lambda}\right) & =\varphi_{j}\left(R^{\prime}\right)\left(x_{\lambda}\right)=\varphi_{l}\left(R^{\prime}\right)\left(x_{\lambda}\right)
\end{aligned}
$$

Hence, for all $i \in N^{\prime}$,

$$
\begin{aligned}
& \varphi_{i}\left(R^{\prime}\right)\left(x_{\lambda}+1\right) \stackrel{(23)}{=} \varphi_{l}\left(R^{\prime}\right)\left(x_{\lambda}+1\right) \\
& \stackrel{(19)}{=} \varphi_{l}(R)\left(\left[x_{\lambda}+1, k\right]\right) \\
& \stackrel{(21)}{=} \varphi_{i}(R)\left(\left[x_{\lambda}+1, p\left(R_{i}\right)\right]\right) \text {. }
\end{aligned}
$$

Furthermore, for all $i \in N^{\prime}$,

$$
\begin{aligned}
& \varphi_{i}\left(R^{\prime}\right)\left(x_{\lambda}\right) \stackrel{(24)}{=} \varphi_{l}\left(R^{\prime}\right)\left(x_{\lambda}\right) \\
& \stackrel{(20)}{=} \varphi_{l}(R)\left(x_{\lambda}\right) \\
& \stackrel{(22)}{=} \varphi_{i}(R)\left(x_{\lambda}\right) \text {. }
\end{aligned}
$$

Since for all $i \in N$ such that $p\left(R_{i}^{\prime}\right) \geq x_{\lambda}+2, \varphi_{i}\left(R^{\prime}\right)\left(\left[x_{\lambda}+2, k\right]\right)=0,(25)$ implies that for all $i \in N^{\prime}$,

$$
\sum_{x_{i}=x_{\lambda}+1}^{k} \varphi_{i}\left(R^{\prime}\right)\left(x_{i}\right) x_{i}=\varphi_{i}\left(R^{\prime}\right)\left(x_{\lambda}+1\right)\left(x_{\lambda}+1\right) \leq \sum_{x_{i}=x_{\lambda}+1}^{k} \varphi_{i}(R)\left(x_{i}\right) x_{i} .
$$

In particular, by (18) and (19),

$$
\varphi_{l}\left(R^{\prime}\right)\left(x_{\lambda}+1\right)\left(x_{\lambda}+1\right)<\sum_{x_{l}=x_{\lambda}+1}^{k} \varphi_{l}(R)\left(x_{l}\right) x_{l} .
$$


Hence, (26), (27), and (28) imply

$$
\sum_{i \in N^{\prime}}\left(\varphi_{i}\left(R^{\prime}\right)\left(x_{\lambda}\right) x_{\lambda}+\varphi_{i}\left(R^{\prime}\right)\left(x_{\lambda}+1\right)\left(x_{\lambda}+1\right)\right)<\sum_{i \in N^{\prime}}\left(\sum_{x_{i}=x_{\lambda}}^{k} \varphi_{i}(R)\left(x_{i}\right) x_{i}\right) .
$$

By Remark 1,

$$
\sum_{i \in N^{\prime}}\left(\varphi_{i}\left(R^{\prime}\right)\left(x_{\lambda}\right) x_{\lambda}+\varphi_{i}\left(R^{\prime}\right)\left(x_{\lambda}+1\right)\left(x_{\lambda}+1\right)\right)=k-\sum_{\substack{i \in N \text { such that } \\ p\left(R_{i}^{\prime}\right)=p\left(R_{i}\right) \leq x_{\lambda}}} p\left(R_{i}\right)
$$

and

$$
\sum_{i \in N^{\prime}}\left(\sum_{x_{i}=x_{\lambda}}^{k} \varphi_{i}(R)\left(x_{i}\right) x_{i}\right)=k-\sum_{\substack{i \in N \text { such that } \\ p\left(R_{i}\right) \leq x_{\lambda}}} p\left(R_{i}\right)
$$

Hence,

$$
\sum_{i \in N^{\prime}}\left(\varphi_{i}\left(R^{\prime}\right)\left(x_{\lambda}\right) x_{\lambda}+\varphi_{i}\left(R^{\prime}\right)\left(x_{\lambda}+1\right)\left(x_{\lambda}+1\right)\right)=\sum_{i \in N^{\prime}}\left(\sum_{x_{i}=x_{\lambda}}^{k} \varphi_{i}(R)\left(x_{i}\right) x_{i}\right),
$$

which contradicts (29). Thus, our assumption that for all $i \in N$ such that $p\left(R_{i}^{\prime}\right) \geq x_{\lambda}+2$ we have $\varphi_{i}\left(R^{\prime}\right)\left(\left[x_{\lambda}+2, k\right]\right)=0$ was wrong. Hence, for some $h \in N, p\left(R_{h}^{\prime}\right) \geq x_{\lambda}+2$ and $\varphi_{h}(R)\left(\left[x_{\lambda}+2, k\right]\right)>0$. Similarly as before, we replace $R_{h}^{\prime}$ by $R_{j}$ and show that for some $m \in N, p\left(R_{m}^{\prime}\right) \geq x_{\lambda}+2$ and $\varphi_{m}\left(R_{-h}^{\prime}, R_{j}\right)\left(\left[x_{\lambda}+2, k\right]\right)>0$. Since $N$ is finite and at each step the number of agents having a peak greater than or equal to $x_{\lambda}+2$ is smaller, we finally get a contradiction. Therefore, (b) holds for Case 1 ; i.e., if $p\left(R_{i}\right) \geq x_{\lambda}+1$, then $\varphi_{i}(R)=U_{i}(R)$.

Case 2: There exists no $j \in N$ such that $p\left(R_{j}\right)=x_{\lambda}+1$ or for $R_{j}$ such that $p\left(R_{j}\right)=x_{\lambda}+1$ there exists some $x_{j} \in\left[p\left(R_{j}\right), k\right]$ such that $B\left(x_{j}, R_{j}\right) \neq\left[p\left(R_{j}\right), x_{j}\right]$.

We have to show (b); i.e., if $p\left(R_{i}\right) \geq x_{\lambda}+1$, then $\varphi_{i}(R)=U_{i}(R)$. First we show that for all $i \in N$ such that $p\left(R_{i}\right) \geq x_{\lambda}+1$,

$$
\varphi_{i}(R)\left(x_{\lambda}\right)=U_{i}(R)\left(x_{\lambda}\right) .
$$

Assume, by contradiction, that for some $j \in N$ such that $p\left(R_{j}\right) \geq x_{\lambda}+1, \varphi_{j}(R)\left(x_{\lambda}\right) \neq$ $U_{j}(R)\left(x_{\lambda}\right)$.

Consider $R_{j}^{\prime} \in \mathcal{R}$ such that $p\left(R_{j}^{\prime}\right)=x_{\lambda}+1$, and for all $x_{j} \in\left[p\left(R_{j}^{\prime}\right), k\right], B\left(x_{j}, R_{j}^{\prime}\right)=$ $\left[p\left(R_{j}^{\prime}\right), x_{j}\right]$. Define $R^{\prime} \equiv\left(R_{j}^{\prime}, R_{-j}\right)$. By strategy-proofness,

$$
\begin{aligned}
\varphi_{j}(R)\left(\left[x_{\lambda}+1, k\right]\right) & =\varphi_{j}(R)\left(B\left(x_{\lambda}+1, R_{j}\right)\right) \\
& \stackrel{S P}{\geq} \varphi_{j}\left(R^{\prime}\right)\left(B\left(x_{\lambda}+1, R_{j}\right)\right)=\varphi_{j}\left(R^{\prime}\right)\left(\left[x_{\lambda}+1, k\right]\right)
\end{aligned}
$$


and

$$
\begin{aligned}
\varphi_{j}\left(R^{\prime}\right)\left(\left[x_{\lambda}+1, k\right]\right) & =\varphi_{j}\left(R^{\prime}\right)\left(B\left(k, R_{j}^{\prime}\right)\right) \\
& \geq \varphi_{j}(R)\left(B\left(k, R_{j}^{\prime}\right)\right) \\
& =\varphi_{j}(R)\left(\left[x_{\lambda}+1, k\right]\right) .
\end{aligned}
$$

Hence, $\varphi_{j}\left(R^{\prime}\right)\left(\left[x_{\lambda}+1, k\right]\right)=\varphi_{j}(R)\left(\left[x_{\lambda}+1, k\right]\right)$. By Lemma 2 ,

$$
\begin{aligned}
\varphi_{j}\left(R^{\prime}\right)\left(x_{\lambda}\right) & =1-\varphi_{j}\left(R^{\prime}\right)\left(\left[x_{\lambda}+1, k\right]\right)=1-\varphi_{j}(R)\left(\left[x_{\lambda}+1, k\right]\right)=\varphi_{j}(R)\left(x_{\lambda}\right) \\
& \neq U_{j}(R)\left(x_{\lambda}\right)=U_{j}\left(R^{\prime}\right)\left(x_{\lambda}\right),
\end{aligned}
$$

which contradicts Case 1. Thus, (30) is proven. Hence, by Lemma 2, for all $i \in N$ such that $p\left(R_{i}\right) \geq x_{\lambda}+1$,

$$
\begin{aligned}
\varphi_{i}(R)\left(\left[x_{\lambda}+1, k\right]\right) & =1-\varphi_{i}(R)\left(x_{\lambda}\right) \\
& =1-U_{i}(R)\left(x_{\lambda}\right) \\
& =U_{i}(R)\left(x_{\lambda}+1\right) .
\end{aligned}
$$

If for all $i \in N$ such that $p\left(R_{i}\right) \geq x_{\lambda}+1, \varphi_{i}(R)\left(\left[x_{\lambda}+2, k\right]\right)=0$, then for all $i \in N$ such that $p\left(R_{i}\right) \geq x_{\lambda}+1, \varphi_{i}(R)=U_{i}(R)$, which proves (b). Suppose, by contradiction, that for some $j \in N$ such that $p\left(R_{j}\right) \geq x_{\lambda}+1, \varphi_{j}(R)\left(\left[x_{\lambda}+2, k\right]\right)>0$. So,

$$
U_{j}(R)\left(x_{\lambda}+1\right)\left(x_{\lambda}+1\right)<\sum_{x_{j}=x_{\lambda}+1}^{k} \varphi_{j}(R)\left(x_{i}\right) x_{i}
$$

Let $N^{\prime} \equiv\left\{i \in N \mid p\left(R_{i}\right) \geq x_{\lambda}+1\right\}$. Since for all $i \in N^{\prime}, U_{i}(R)\left(\left[x_{\lambda}+2, k\right]\right)=0$,

$$
U_{i}(R)\left(x_{\lambda}+1\right)\left(x_{\lambda}+1\right) \leq \sum_{x_{i}=x_{\lambda}+1}^{k} \varphi_{i}(R)\left(x_{i}\right) x_{i} .
$$

Hence, by (30), (31), and (32),

$$
\sum_{i \in N^{\prime}}\left(U_{i}(R)\left(x_{\lambda}\right) x_{\lambda}+U_{j}(R)\left(x_{\lambda}+1\right)\left(x_{\lambda}+1\right)\right)<\sum_{i \in N^{\prime}}\left(\sum_{x_{i}=x_{\lambda}}^{k} \varphi_{i}(R)\left(x_{i}\right) x_{i}\right) .
$$

By Remark 1,

$$
\sum_{i \in N^{\prime}}\left(\sum_{x_{i}=x_{\lambda}}^{k} \varphi_{i}(R)\left(x_{i}\right) x_{i}\right)=k-\sum_{\substack{i \in N \text { such that } \\ p\left(R_{i}\right) \leq x_{\lambda}}} p\left(R_{i}\right)
$$


and

$$
\sum_{i \in N^{\prime}}\left(U_{i}(R)\left(x_{\lambda}\right) x_{\lambda}+U_{i}(R)\left(x_{\lambda}+1\right)\left(x_{\lambda}+1\right)\right)=k-\sum_{\substack{i \in N \text { such that } \\ p\left(R_{i}\right) \leq x_{\lambda}}} p\left(R_{i}\right) .
$$

Hence,

$$
\sum_{i \in N^{\prime}}\left(U_{i}(R)\left(x_{\lambda}\right) x_{\lambda}+U_{i}(R)\left(x_{\lambda}+1\right)\left(x_{\lambda}+1\right)\right)=\sum_{i \in N^{\prime}}\left(\sum_{x_{i}=x_{\lambda}}^{k} \varphi_{i}(R)\left(x_{i}\right) x_{i}\right),
$$

which contradicts (33) and finishes the proof of (b); i.e., if $p\left(R_{i}\right) \geq x_{\lambda}+1$, then $\varphi_{i}(R)=$ $U_{i}(R)$.

\section{Von-Neumann-Morgenstern Utility Functions}

In this section we assume that agents have single-peaked preferences that satisfy the von Neuman Morgenstern (vNM) expected utility property; i.e., for any preference relation $R_{i}$

(i) there exists a utility function $u_{i}$ that represents $R_{i}$ and $^{5}$

(ii) each agent $i$ evaluates marginal distributions via the expected utility relative to $u_{i}$. Thus, given $Q_{i} \equiv\left[p_{i}^{0} \circ 0, p_{i}^{1} \circ 1, \ldots, p_{i}^{k} \circ k\right]$ where $p_{i}^{l}$ denotes the probability that agent $i$ receives $l \in K$ objects,

$$
u_{i}\left(Q_{i}\right)=u_{i}\left(\left[p_{i}^{0} \circ 0, p_{i}^{1} \circ 1, \ldots, p_{i}^{k} \circ k\right]\right)=\sum_{l=0}^{k} p_{i}^{l} u_{i}(l) .
$$

Furthermore, we assume that all agents are strictly risk averse; i.e., for all $l \in\{1, \ldots, k-$ $1\}$,

$$
u_{i}(l)-u_{i}(l-1)>u_{i}(l+1)-u_{i}(l)
$$

Let $\mathcal{V}$ denote the class of all vNM-utility functions that exhibit strict risk aversion. Let $\mathcal{V}^{N}$ denote the set of all (vNM-utility) profiles $u=\left(u_{i}\right)_{i \in N}$ such that for all $i \in N, u_{i} \in \mathcal{V}$. Note that if a utility function exhibits strict risk aversion, then it is single-peaked. Given

\footnotetext{
${ }^{5}$ A utility function $u_{i}$ represents a preference relation $R_{i}$ if and only if

$$
x R_{i} y \Leftrightarrow u_{i}(x) \geq u_{i}(y)
$$
}


$u_{i} \in \mathcal{V}$, there exists $R_{i} \in \mathcal{R}$ such that $u_{i}$ represents $R_{i}$. We define the peak $p\left(u_{i}\right)$ of $u_{i}$ by $p\left(u_{i}\right) \equiv p\left(R_{i}\right)$. Let $\overline{\mathcal{R}}$ denote the set of single-peaked preference relations such that there exists some $u_{i} \in \mathcal{V}$ that represents $R_{i}$. Strict risk aversion implies that $\overline{\mathcal{R}} \subsetneq \mathcal{R}$.

Let $\mathcal{D}(X)$ denote the set of all distributions over $X$. Throughout this section a probabilistic rule is a function $\varphi: \mathcal{V}^{N} \rightarrow \mathcal{D}(X)$. Next, we reformulate our central properties by replacing preference relations by utility functions.

Let $Q, Q^{\prime}$ be distributions over $X$. If for all $i \in N, u_{i}\left(Q_{i}\right) \geq u_{i}\left(Q_{i}^{\prime}\right)$ and for some $j \in N$, $u_{j}\left(Q_{j}\right)>u_{j}\left(Q_{j}^{\prime}\right)$, then we call $Q$ a Pareto improvement of $Q^{\prime}$.

Pareto Efficiency: For all $u \in \mathcal{V}^{N}$, there exists no Pareto improvement of $\varphi(R)$.

Given $u \in \mathcal{V}^{N}$ and $M \subseteq N$, the restriction $\left(u_{i}\right)_{i \in M} \in \mathcal{V}^{M}$ of $u$ to $M$ is denoted by $u_{M}$. Similarly as before, $\left(\bar{u}_{i}, u_{-i}\right)$ denotes the profile obtained from $u$ by replacing $u_{i}$ by $\bar{u}_{i}$.

Strategy-Proofness: $\quad$ For all $u \in \mathcal{V}^{N}$, all $i \in N$, and all $\bar{u}_{i} \in \mathcal{V}, u_{i}\left(\varphi_{i}(u)\right) \geq u_{i}\left(\varphi_{i}\left(\bar{u}_{i}, u_{-i}\right)\right)$.

For all $u \in \mathcal{V}^{N}$ and all $\pi \in \Pi^{N}$, by $u_{\pi}$ we mean $\left(u_{\pi(i)}\right)_{i \in N}$.

Anonymity: For all $u \in \mathcal{V}^{N}$, all $\pi \in \Pi^{N}$, and all $i \in N, \varphi_{\pi(i)}(u)=\varphi_{i}\left(u_{\pi}\right)$.

No-Envy: For all $u \in \mathcal{V}^{N}$ and all $i, j \in N, u_{i}\left(\varphi_{i}(R)\right) \geq u_{i}\left(\varphi_{j}(R)\right)$.

Equal Treatment of Equals: For all $u \in \mathcal{V}^{N}$ and all $i, j \in N$, if $u_{i}=u_{j}$, then $u_{i}\left(\varphi_{i}(u)\right)=u_{i}\left(\varphi_{j}(u)\right)$.

Using strictly risk averse vNM-utility functions induces a stronger Pareto efficiency condition than using the ordinal preference extension. Same-sidedness is still implied, but no longer sufficient. In order to characterize Pareto efficiency we introduce the following notation.

Let $Q$ be a distribution and for all $i \in N, Q_{i}=\left[p_{i}^{0} \circ 0, p_{i}^{1} \circ 1, \ldots, p_{i}^{k} \circ k\right]$ denotes the induced marginal distribution. For all $i \in N$, let

$$
G_{i}\left(Q_{i}\right)=\left\{l \in K \mid p_{i}^{l} \neq 0\right\} .
$$

Hence, $G_{i}\left(Q_{i}\right)$ denotes all amounts that agent $i$ can possibly receive at $Q$ (or equivalently, $G_{i}\left(Q_{i}\right)$ is the support of $\left.Q_{i}\right)$. 
The following Lemma is due to Sasaki (1997), Lemma 6.3.

Lemma 3 (Sasaki, 1997) If a probabilistic rule $\varphi: \mathcal{V}^{N} \rightarrow \mathcal{D}(X)$ satisfies Pareto efficiency on $\mathcal{V}^{N}$, then for all $u \in \mathcal{V}^{N}$ and all $i \in N$ there exists a number of objects $a_{i} \in K$ such that $G_{i}\left(\varphi_{i}(u)\right) \subseteq\left\{a_{i}, a_{i}+1\right\}$.

Using Lemma 3, we can derive a characterization of Pareto efficiency on $\mathcal{V}^{N}$.

Lemma 4 A probabilistic rule $\varphi: \mathcal{V}^{N} \rightarrow \mathcal{D}(X)$ satisfies Pareto efficiency on $\mathcal{V}^{N}$ if and only if

(i) $\varphi$ satisfies ex-post Pareto efficiency on $\mathcal{V}^{N}$ and

(ii) for all $u \in \mathcal{V}^{N}$ and all $i \in N$ there exists a number of objects $a_{i} \in K$ such that $G_{i}\left(\varphi_{i}(u)\right) \subseteq\left\{a_{i}, a_{i}+1\right\}$.

The only-if part of Lemma 4 follows from Lemma 3. We omit the proof of the if-part of Lemma $4 .^{6}$

Lemma 4 implies that any Pareto efficient allocation can be represented as a vector of nonnegative real numbers. Let $Q$ be a distribution that is Pareto efficient for some $u \in \mathcal{V}^{N}$. Then $Q$ can be represented as follows. According to Lemma 4, for all $i \in N$ there exists a number of objects $a_{i} \in K$ such that $G_{i}\left(Q_{i}\right) \subseteq\left\{a_{i}, a_{i}+1\right\}$. By $\mu_{i}$ we denote the probability at $Q_{i}$ for agent $i$ to receive $a_{i}$. Thus, agent $i$ receives $a_{i}+1$ at $Q_{i}$ with probability $1-\mu_{i}$. Using these unique probabilities, we can represent the distribution $Q_{i}$ by a unique number $q_{i} \in[0, k]$. Simply define

$$
q_{i} \equiv \mu_{i} a_{i}+\left(1-\mu_{i}\right)\left(a_{i}+1\right)=\left(a_{i}+1\right)-\mu_{i}
$$

Note that we have $\sum_{i \in N} q_{i}=k$. Thus, each distribution $Q$ is identified with a unique allocation $\left(q_{1}, \ldots, q_{n}\right) \in[0, k]^{N}$ of $k$ units of an infinitely divisible commodity. Let $\tilde{\mathcal{D}}(X)$ denote the set of all distributions $Q \in \mathcal{D}(X)$ such that for all $i \in N$ there exists some $a_{i} \in K$ for which $G_{i}\left(Q_{i}\right) \subseteq\left\{a_{i}, a_{i}+1\right\}$. Hence, a Pareto efficient probabilistic rule is a function $\varphi: \mathcal{V}^{N} \rightarrow \tilde{\mathcal{D}}(X)$. Let $\mathcal{X}(N, k)$ denote the set of all allocations that are obtained via some distribution belonging to $\tilde{\mathcal{D}}(X)$, i.e., $\mathcal{X}(N, k) \equiv\left\{\left(q_{1}, \ldots, q_{n}\right) \mid Q \in \tilde{\mathcal{D}}(X)\right\}$. Obviously, $X \subsetneq$ $\mathcal{X}(N, k)$. Therefore, a Pareto efficient rule $\varphi$ essentially splits the amount $k$ of a perfectly divisible good among the agents in $N$. Conversely, each vector $\left(q_{1}, \ldots, q_{n}\right) \in \mathcal{X}(N, k)$

\footnotetext{
${ }^{6}$ The somewhat tedious proof of the if-part of Lemma 4 is available from the authors upon request.
} 
uniquely identifies marginal distributions $Q_{i}$ and a (non-unique) distribution $Q \in \tilde{\mathcal{D}}(X)$. Note that single-peakedness on $K$ implies single-peakedness on $[0, k]$ if agents consider assignments $q_{i} \in[0, k]$ that correspond to marginal distributions $Q_{i}$. Therefore, the problem of assigning $k$ identical objects to a set of agents with single-peaked preferences by using a Pareto efficient probabilistic rule reduces to the problem of dividing the amount $k$ of a perfectly divisible homogeneous commodity among a group of agents with single-peaked preferences.

So, any Pareto efficient probabilistic rule $\varphi: \mathcal{V}^{N} \rightarrow \tilde{\mathcal{D}}(X)$ induces a Pareto efficient deterministic allocation rule $\Phi: \overline{\mathcal{R}}^{N} \rightarrow \mathcal{X}(N, k)$ for the problem of dividing the amount $k$ of a perfectly divisible homogeneous commodity among a group of agents with single-peaked preferences. Furthermore, if the probabilistic rule also satisfies strategy-proofness and noenvy, then the induced allocation rule satisfies these properties as well. Any probabilistic rule with uniform marginal distributions induces the uniform allocation rule and vice versa. ${ }^{7}$

The domain $\overline{\mathcal{R}}$ satisfies the following "richness condition": given $x_{i}, y_{i} \in[0, k]$, if ]$x_{i}, y_{i}\left[\cap K \neq \emptyset\right.$, then for all $\left.z_{i} \in\right] x_{i}, y_{i}\left[\cap K\right.$ there exist preference relations $R_{i}, \bar{R}_{i} \in \overline{\mathcal{R}}$ such that $p\left(R_{i}\right)=p\left(\bar{R}_{i}\right)=z_{i}, x_{i} P_{i} y_{i}$, and $y_{i} P_{i} x_{i}$. It can be checked that for example for the proofs of Ching (1994) this condition suffices to show that if a deterministic rule $\Phi: \overline{\mathcal{R}}^{N} \rightarrow \mathcal{X}(N, k)$ satisfies same-sidedness, strategy-proofness, and equal treatment of equals, then it is the uniform rule restricted on this domain.

All characterization results obtained for Pareto efficient deterministic rules $\Phi: \overline{\mathcal{R}}^{N} \rightarrow$ $\mathcal{X}(N, k)$ that divide a perfectly divisible homogeneous commodity among a group of agents with single-peaked preferences also hold for probabilistic rules that assign indivisible identical objects to a set of strictly risk-averse agents with single-peaked preferences. Since the marginal distributions of the uniform probabilistic rules in Section 4 only depend on the peak profile, we define these rules in the same way in the current context for profiles of utility functions.

Corollary 1 (Kureishi, 2000) When each agent is a strictly risk averse vNM-expected utility maximizer, the uniform probabilistic rules are the only probabilistic rules satisfying Pareto efficiency, strategy-proofness, and equal treatment of equals.

\footnotetext{
${ }^{7}$ Uniform Allocation Rule $\tilde{U}:$ For all $R \in \overline{\mathcal{R}}^{N}$, and all $j \in N$,

$$
\tilde{U}_{j}(R) \equiv \begin{cases}\min \left(p\left(R_{j}\right), \lambda\right) & \text { if } \sum_{N} p\left(R_{i}\right) \geq k \\ \max \left(p\left(R_{j}\right), \lambda\right) & \text { if } \sum_{N} p\left(R_{i}\right) \leq k\end{cases}
$$
}

where $\lambda$ solves $\sum_{N} \tilde{U}_{i}(R)=k$. 
Since equal treatment of equals is implied by either anonymity or no-envy, in Corollary 1 we can replace equal treatment of equals either by anonymity (Sasaki, 1997) or by no-envy.

\section{References}

Abdulkadiroğlu, A., and T. Sönmez (1998): Random Serial Dictatorship and the Core from Random Endowments in House Allocation Problems, Econometrica 66:689-701.

Abdulkadiroğlu, A., and T. Sönmez (2000): Ordinal Efficiency and Dominated Sets of Assignments, Working Paper, Northwestern University.

Benassy, J.P. (1982): The Economics of Market Disequilibrium, San Diego: Academic Press.

Bogomolnaia, A., and H. Moulin (1999): A New Solution to the Random Assignment Problem, Journal of Economic Theory, forthcoming.

Ching, S. (1992): A Simple Characterization of the Uniform Rule, Economics Letters 40:57-60.

Ching, S. (1994): An Alternative Characterization of the Uniform Rule, Social Choice and Welfare 11:131-136.

Crès, H., and H. Moulin (1998): Random Priority: A Probabilistic Resolution of the Tragedy of the Commons, Working Paper, Duke University.

Ehlers, L. (1998): Probabilistic Allocation Rules and Single-Dipped Preferences, Social Choice and Welfare, forthcoming.

Ehlers, L. (2000): Indifference and the Uniform Rule, Economics Letters 67:303-308.

Ehlers, L., and B. Klaus (2001): Solidarity and Probabilistic Target Rules, Journal of Public Economic Theory, 3:167-184.

Ehlers, L., H. Peters, and T. Storcken (2000): Strategy-Proof Probabilistic Decision Schemes for One-Dimensional Single-Peaked Preferences, Journal of Economic Theory, forthcoming.

Gibbard, A. (1977): Manipulation of Schemes that Mix Voting and Chance, Econometrica 45:665-681. 
Kureishi, W. (2000): A Simple Characterization of the Randomized Uniform Rule, Working Paper, Osaka University.

Moulin, H. (2000): The Proportional Random Allocation of Indivisible Units, Social Choice and Welfare, forthcoming.

Moulin, H., and R. Stong (2000): Fair Queuing and Other Probabilistic Allocation Methods, Working Paper, Rice University.

Sasaki, H. (1997): Randomized Uniform Allocation Mechanism and Single-Peaked Preferences if Indivisible Good, Working Paper, Waseda University.

Sprumont, Y. (1991): The Division Problem with Single-Peaked Preferences: A Characterization of the Uniform Rule, Econometrica 59:509-519. 